Actes du 17e colloque du GERAS

\title{
Cohérences plurielles
}

Jean Sabiron

\section{OpenEdition \\ Journals}

Édition électronique

URL : http://journals.openedition.org/asp/3537

DOI : 10.4000/asp.3537

ISSN : 2108-6354

\section{Éditeur}

Groupe d'étude et de recherche en anglais de spécialité

\section{Édition imprimée}

Date de publication : 1 décembre 1996

Pagination : 239-252

ISSN : 1246-8185

Référence électronique

Jean Sabiron, « Cohérences plurielles », ASp [En ligne], 11-14 | 1996, mis en ligne le 09 mai 2013,

consulté le 19 avril 2019. URL : http://journals.openedition.org/asp/3537 ; DOI : 10.4000/asp.3537

Ce document a été généré automatiquement le 19 avril 2019.

Tous droits réservés 


\title{
Cohérences plurielles
}

\author{
Jean Sabiron
}

1 L'objet de la présente communication n'est pas la présentation d'un paradigme globalisant ou d'une méthodologie centralisante, unitaire, voire idéologique, mais celle des grands traits d'une combinatoire d'outils langagiers et méthodologiques pour un public de DEUG scientifique.

2 Dans ce paradigme de l'innovation qui supplante celui d'une méthodologie unique, je présente ici des "cohérences locales, temporelles et plurielles", selon les propos de C. Puren (1994), à l'usage des grands nombres.

3 Je me situe ainsi en didacticien, c'est-à-dire dans le cadre d'une science autonome dont la réflexion essentiellement psychologique et épistémologique permet de fonder des pratiques pédagogiques sur une approche rationnelle de tous les mécanismes en jeu dans la transmission des connaissances. Je revendique ainsi les influences et fondements théoriques suivants: le constructivisme piagétien, la psycholinguistique et la pragmatique, ainsi que les sciences cognitives, dont les neurosciences.

4 En direction du public de DEUG Sciences, je vise donc à cohérer dans un projet social l'appropriation d'un certain savoir langagier, d'une culture procédurale, et de l'étape métacognitive d'un savoir-être. La visée finale du processus engagé est l'autonomisation de l'étudiant à l'université, par le transfert progressif de la prise de décision dans l'apprentissage, de l'institution à l'apprenant adulte.

5 Le public étudiant est celui principalement du DEUG B2 Sciences de la Vie et de la Terre, (8 groupes de 30 à 40 étudiants), 1'enseignant titulaire et quatre vacataires; $50 \mathrm{~h}$ d'enseignement par an, pas d'anglais en $1^{\text {re }}$ année, d'où en réalité une interruption moyenne de 27 mois.

6 Les trois objectifs annoncés sont les suivants :

- réactiver les connaissances et les procédures en sommeil, par une multitude de tâches successives mettant en œuvre les compétences de compréhension et production orales, et celle de la lecture.

- organiser ses connaissances en vue d'acquérir une méthodologie critique universitaire. 
- enfin, en $3^{\mathrm{e}}$ lieu seulement, acquérir et manipuler les connaissances plus spécifiquement nécessaires à l'étudiant scientifique de second et troisième cycle, en ce qui concerne le lexique et les principales fonctions du discours scientifique.

7 Trois niveaux de cohérence seront proposés : un premier, de nature langagière, un second, de nature procédurale, et le dernier, de nature anthropologique.

\section{La cohérence dans la réactivation d'un savoir langagier}

Après une aussi longue interruption d'activités de langues, les étudiants se voient proposer une alternance d'activités présentielles puis en autonomie préparée, autour d'études sur une période de deux ou trois semaines de thématiques ou plutôt de problématiques, c'est-à-dire de situations pour lesquelles il n'existe pas encore de solution ou de réponses. Ces sujets peuvent être "scientifiques » - animaux en captivité, liaison ou non entre lignes à haute tension et cancers - mais cela n'est pas une nécessité (cf. la réalité viticole en Grande-Bretagne)

Figure 1.Tableau du découpage annuel

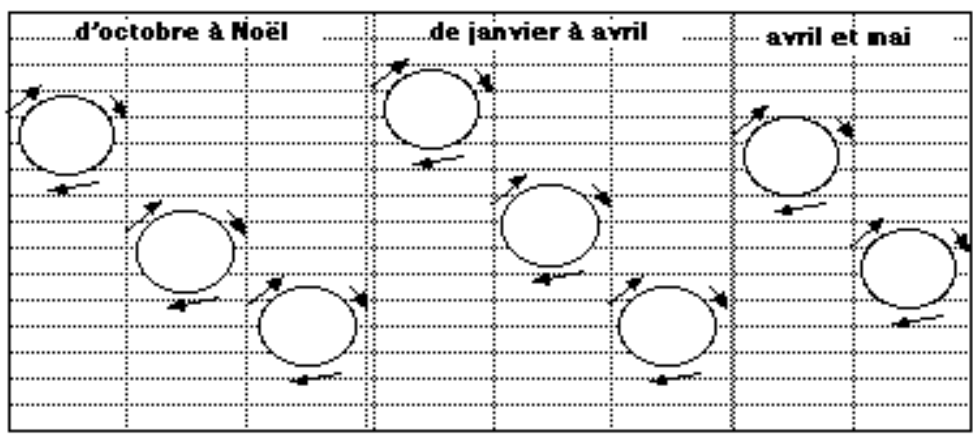

Les trois compétences privilégiées sont celles de compréhension orale et écrite, avec une insistance particulière sur la compréhension de l'oral, "l'aptitude oubliée », selon Holec, et celle de la production orale spontanée (Figure 1). Pour le schéma détaillé d'un des cycles de trois semaines, voir figure complémentaire en annexe 1.

À titre d'illustration, voici la mise en œuvre des outils langagiers proposés en vue du perfectionnement de la compréhension orale.

\subsection{Phase 1}

11 La phase 1, présentielle se déroule selon la progression validée par la plupart des didacticiens: une construction progressive du sens de documents télévisuels authentiques (en l'occurrence des séquences de bulletins d'informations de SkyNews et $\mathrm{CNN}$, préalablement sélectionnés et «didactisés » par l'équipe VIFAX ${ }^{1}$ ) par des étapes successives d'anticipation, seul puis à deux à partir du titre, puis la prise d'indices de l'image muette, des liens de causalité plausibles entre les séquences, donc de la « mise en texte » ou en image de l'auteur du document.

Dans cette première cohérence, sont mises en œuvre des opérations mentales de haut niveau. Amorçage conscient, focalisation, activation de schémas ou nœuds du réseau 
sémantique et anticipation constituent la démarche onomasiologique, c'est-à-dire celle qui conduit du concept au stimulus.

13 L'anticipation est une activité linguistique à part entière et elle a une valeur sociale, d'interactivité, de confrontation des hypothèses de sens et des intentions du locuteur; elle mène à un conflit socio-cognitif, à une co-élaboration, une émulation, et à une motivation. Elle fait en outre établir des liens et manipuler des concepts et « des mots pour le dire». Dans cette phase, les étudiants mettent en œuvre une procédure de résolution partielle d'un problème: seuls ou à deux, ils doivent reconstituer l'enchaînement causal et la présomption de pertinence voulus par les auteurs du message.

14 À la fin de cette phase, les principales hypothèses sont vérifiées, par la reconstitution collective de l'intégralité du contenu de l'introduction du message sonore, ceci pour éviter la dispersion dans l'interprétation du reste du document. Mes expériences ont montré qu'il faut partiellement clôturer le sens pour éviter une surcharge cognitive pendant la tâche suivante, d'écoute hors établissement.

\subsection{Phase 2}

La $2^{\mathrm{e}}$ phase a lieu hors établissement. Chaque étudiant reçoit sur cassette audio une copie de la bande-son du document. Cette appropriation de l'outil, du document, et la gestion autonome du temps d'écoute individuelle et de la lecture extensive d'accompagnement est ergonomique: l'auditeur a besoin de moins de prises d'indices pour valider ses hypothèses de sens.

Dans cette tâche individuelle guidée, d'exploration, d'observation, de repérage, de segmentation, de reconnaissance des redondances escomptées, la première interprétation de sens du message répond au principe de pertinence. Ceci permet une meilleure disposition attentionnelle pour l'identification de l'inattendu sémantique, des articulateurs du discours, des schémas intonatoires, entre autres, et surtout pour les inférences : bref pour le repérage de ces représentations pertinentes qui vont « au-delà de ce qui est explicitement affirmé » selon les termes de Johnson-Laird (1980).

Les matériaux didactisés par VIFAX sont reconditionnés et complétés pour ce public de DEUG (annexe 2). La réception par modem des documents VIFAX permet de traiter physiquement les scripts pour, par exemple, mettre en évidence les mots répertoriés par J. Upjohn et alii (1991), les 645 mots qui me servent de référence constante (cf. annexe 3).

18 Dans cette phase de travail individuel, l'étudiant dispose aussi de la feuille ACADEME .D'une façon générale, l'auditeur non natif n'a pas suffisamment de connaissances lexicales, conceptuelles et/ou culturelles pour réaliser son objectif d'écoute. Donc, nous fournissons, sous le nom d'ACADEME, le contexte référentiel, linguistique, conceptuel et pragmatique de l'énoncé oral à comprendre, sous la forme d'une feuille A4 par thématique (cf. annexes 4 et 5)

19 En somme, cet apport isotopique, cette continuité du thème traité, réduisent «les asymétries entre interlocuteurs », dit Armengaud (1985). Ne pas fournir un minimum de connaissances à un auditeur non natif relèverait de l'incohérence.

20 Cette feuille de guidage encourage l'heuristique, et facilite l'interprétation, et ainsi, l'économie dans l'attention sélective qu'elle entraîne dans la tâche d'écoute. 
21 En conclusion, l'approche langagière a la cohérence d'un savoir construit, et d'une signification progressivement attribuée, puis affinée, et enfin dépassée.

La démarche onomasiologique, en compréhension, convient bien à l'approche scientifique d'un savoir car elle est très proche de la méthode hypothético-déductive en science expérimentale. Dans les deux cas, le sujet fait des hypothèses et il les vérifie ; la relation causale est le moteur de la découverte et du raisonnement scientifique. Plus les hypothèses de sens ou de phénomènes auront été riches, plus la tâche d'écoute ou d'observation sera une vérification de l'attendu. « ... il faut comprendre que le microscope est un prolongement de l'esprit plutôt que de l'œil » écrit Bachelard (1938).

Apparaît ici une première cohérence ou convergence entre la didactique des langues et l'esprit scientifique et donc la didactique des sciences. Pour Jean Caron, le psycholinguiste, "un discours est cohérent si ses éléments successifs peuvent être intégrés en une représentation unitaire» (1992). La cohérence dépasse donc le cloisonnement disciplinaire.

24 Le temps ainsi libéré par des tâches d'audition, de vérification, de lectures extensives hors établissement, permet de consacrer plus de temps in situ à la méthodologie de l'organisation ou de la structuration des savoirs, et des rattachements des savoirs nouveaux aux savoirs antérieurs en vue d'accroître les schèmes de références et les capacités de mémorisation. Le savoir langagier ne représente qu'un tiers de notre temps de travail.

\section{La méthodologie du savoir-faire, une autre phase présentielle}

Pour Gaonac'h (1987), est cognitive une activité «conduisant à la construction d'une connaissance organisée de l'environnement». Et l'auteur ajoute, en substance, que la structure est moins à chercher dans l'objet linguistique que dans l'activité. Celle-ci est le facteur prépondérant de l'acquisition.

L'anticipation et les autres opérations de haut niveau qui ont lieu dans la première phase de la compréhension sont des opérations de construction préalables aux stimuli ou énoncés. Mais cette discipline scientifique suppose des outils intellectuels performants. Plus notre capacité de traitement et d'interprétation des connaissances est efficace et automatisée, plus notre réponse à une situation nouvelle sera pertinente et rapide.

Catégorisation, analogies, et conceptualisation sont les mots clés d'une intériorisation de l'environnement qui aboutit à une formation de représentations ou de cartes cognitives.

Après trois ans d'expérimentation en DEUG, je soutiendrai volontiers qu' « analyser le réel en termes d'objet, de propriétés et relations " selon les termes de Vergnaud (in Grand dictionnaire de la psychologie, Paris : Larousse, 1993), est à la base de la construction d'un esprit critique universitaire. Le DEUG est le moment privilégié pour ces activités procédurales et comportementales

Désormais, nous passons autant de temps à structurer et interpréter les connaissances qu'à en acquérir.

Sont ainsi privilégiés trois types d'exercices procéduraux :

- - la définition : il ne s'agit plus d'inclure un énoncé dans une situation mais de le délier des conditions de l'énonciation et de retrouver le concept d'appartenance, 
- - la classification (différente du classement),

- - et la comparaison : analogies et contrastes.

Cette activité du traitement de l'information, de nature computationnelle et connexionniste, s'apparente à une démarche neuronale faite d'activations et d'inhibitions. Il s'agit bien de la restructuration continuelle préconisée par Mc Laughlin (1987), telle qu'elle intervient dans chaque cycle d'études.

31 Une telle routine procédurale s'apparente à l'esprit critique scientifique et à une méthode scientifique de l'acquisition des connaissances, il s'agit donc ici d'un autre niveau de cohérence: il y a bien une convergence entre la didactique des langues et celles des sciences. J'en extrais les aspects suivants :

32 - les ancrages cognitifs et les références aux opérations mentales. Les didacticiens en sciences et en mathématiques puisent à des sources qui nous sont communes. "Les hypothèses constructivistes sont très majoritairement retenues ", écrivent Joshua et Dupin. De Vecchi et Giordan parlent d'heuristique, de schèmes comportementaux, de pragmatique, et tiennent des propos culioliens.

33 - des liens étroits existent entre l'onomasiologie et approche hypothético-déductive. Bachelard est proche de Claparède, et de la logique propositionnelle de Piaget.

34 - en matière d'apprentissage, l'accent est mis sur l'organisation des connaissances et la procéduralisation. La méthodologie expérimentale est un processus permanent de restructuration des connaissances; "le savoir de l'expert est plus organisé, mis en structure et fortement hiérarchisé » (Joshua 1993).

35 - il existe de nombreuses convergences entre la modélisation scientifique et les notions de conceptualisation et d'abstraction exposées par B.M. Barth (1987).

36 - dans le domaine des sciences et dans celui des langues pour un public LANSAD ${ }^{2}$ il y a d'autre part une prédominance de discours descriptifs, explicatifs, et argumentatifs. La référence partagée à l'architecture cognitive présentée par J.-F. Richard (1990) nous invite à approfondir les opérations de raisonnement, les concepts de repères ou d'invariants fondamentaux, l'importance des stratégies de similarité, et les relations de causalité, d'effet et de conséquence. De nombreuses convergences complémentaires peuvent émerger de la lecture d'Astolfi, Balacheff, Brousseau, Chevallard, Douady, Lakatos...

38 La connaissance de l'approche didactique des disciplines autres que la langue étrangère est essentielle pour le didacticien en langues en milieu LANSAD, et ce d'un point de vue tant épistémologique que pragmatique. Il nous appartient, au GERAS, d'assurer la diffusion ou la propagation de ces convergences. Une cohérence interdisciplinaire est à construire. La science, dit Le Petit Larousse est « un ensemble cohérent de connaissances ». Le didacticien en langue peut contribuer à cette cohérence, à cette intégration. Je fais mienne la critique de la parcellisation du savoir que fait souvent M. Perrin : elle rejoint celle d'Edgard Morin (1991) :

On arrive à une clôture disciplinaire, hyper-disciplinaire, où chacun évidemment est propriétaire d'un maigre territoire et compense son incapacité à réfléchir sur les territoires des autres par l'interdiction rigoureuse faite à autrui de pénétrer sur le sien. 


\section{Vers la cohérence anthropologique}

39 la notion de combinatoire d'outils langagiers et de méthodes procédurales. Le didacticien crée des articulations cohérentes successives selon les groupes, selon le rythme de vie des capacités des étudiants et des périodes de l'année universitaire, et prévoit des activités de rupture.

La visée intégrative que nous poursuivons devra toujours se prévaloir « d'une pensée et d'une activité qui remembrent et relient" (Bougnoux 1993). À tout moment de l'acquisition, l'apprenant doit faire le point pour rattacher les connaissances nouvelles aux schémas existants. Cette nécessité est inhérente à l'apprentissage, elle est aussi essentielle à l'homme. Cette exigence topologique est inhérente à sa nature.

La topologie est un espace de signes, une cartographie. Tout mouvement permet un remodelage $\mathrm{du}$ réseau de relations ou de rapports aux repères. Les outils méthodologiques de catégorisation et d'abstraction expérimentés participent à la structuration de repères et d'invariants. Ils préparent une navigation autonome en fournissant les limites ou les proximités.

41 À sa façon, la feuille ACADEME, modeste portulan, contribue à cette cartographie, en signalant les contours, en suggérant des escales; elle est à la fois un ancrage essentiel et un appel à la «navigation ». Son but est bien d'inviter l'étudiant à explorer les sources de références mentionnées dans chaque feuille, aujourd'hui sur support papier, et demain sur support numérique.

qu'advient-il de la linéarité de nos acquisitions et de notre culture face à l'hypernavigation et l'aléatoire décontextualisé du cédérom et du WEB ?

\section{Conclusion}

Quels que soient les thèmes abordés, les modes opératoires restent les mêmes : le savoir se construit, le sens est attribué, la connaissance réorganisée.

Au moment où le DEUG Sciences devient spécialisé et perd son caractère d'études générales, et où les savoirs des spécialistes scientifiques présentés aux étudiants de ce niveau sont de plus en plus fragmentés et cloisonnés, il est vital de faire des exercices de repérage des entités organisatrices et des convergences transversales.

46 Le risque actuel est l'accélération de l'atomisation des savoirs, renforcée par les supports numériques qui les dispensent de façon spontanée et massive. Il y a un danger d'errance, ou de perte des repères ontologiques fondamentaux que sont l'appartenance à un « territoire ", et la notion du temps.

4 Dans le cadre des activités de langues en DEUG Sciences, nous, didacticiens de langues, pouvons contribuer à ce que l'étudiant ne navigue pas, ou n'hypernavigue pas, sans boussole.

Chaque fois qu'un être humain organise ou réorganise son rapport à lui-même, à ses semblables, aux choses, aux signes, au cosmos, il est engagé dans une activité de connaissance, d'apprentissage. (Lévy 1994) 


\section{BIBLIOGRAPHIE}

Armengaud, F. 1985. La pragmatique. Paris : Presses universitaires de France.

Bachelard, G. 1938. La formation de l'esprit scientifique. Paris : Vrin.

Barth, B.M. L'apprentissage de l'abstraction, Paris : Retz, 1987.

Bougnoux, D. Sciences de l'information et de la communication : textes essentiels, Paris : Larousse, 1993.

De Vecchi, G. et A. Giordan. 1994. L'enseignement scientifique. Nice : Z'éditions.

Johnson-Laird, P.N. 1980. « Modèles mentaux en sciences cognitives ». Bulletin de psychologie 51383, traduction d'un article paru dans Cognitive Science.

Joshua, S. et J.-J. 1993. Dupin. Introduction à la didactique des sciences et des mathématiques. Paris : Presses universitaires de France.

Lévy, P. 1994. L'intelligence collective. Pour une anthropologie du cyberspace. Paris : La Découverte. Mc Laughlin, B. 1987. Theories of Second-Language Learning. Londres : Hooder and Stoughton. Morin, E. 1991. La Méthode. 4 : Les idées. Leur habitat, leur vie, leurs mœurs, leur organisation. Paris : Le Seuil.

Perrin, M. 1982. «Cohérence dans l'éclectisme : l'andragogie des langues pour la décennie à venir ». Formation France, janvier, 12-15.

Puren, C. 1994. La didactique des langues étrangères à la croisée des méthodes. Essai sur l'éclectisme. Paris : Didier.

Richard, J.-F. 1990. Les activités mentales. Comprendre, raisonner, trouver des solutions. Paris : A. Colin. Upjohn, J., S. Blattes et V. Jans. 1991. Minimum Competence in Scientific English. Grenoble : Presses universitaires de Grenoble.

\section{ANNEXES}

\section{Annexe 1}

\section{Cycle de trois semaines}




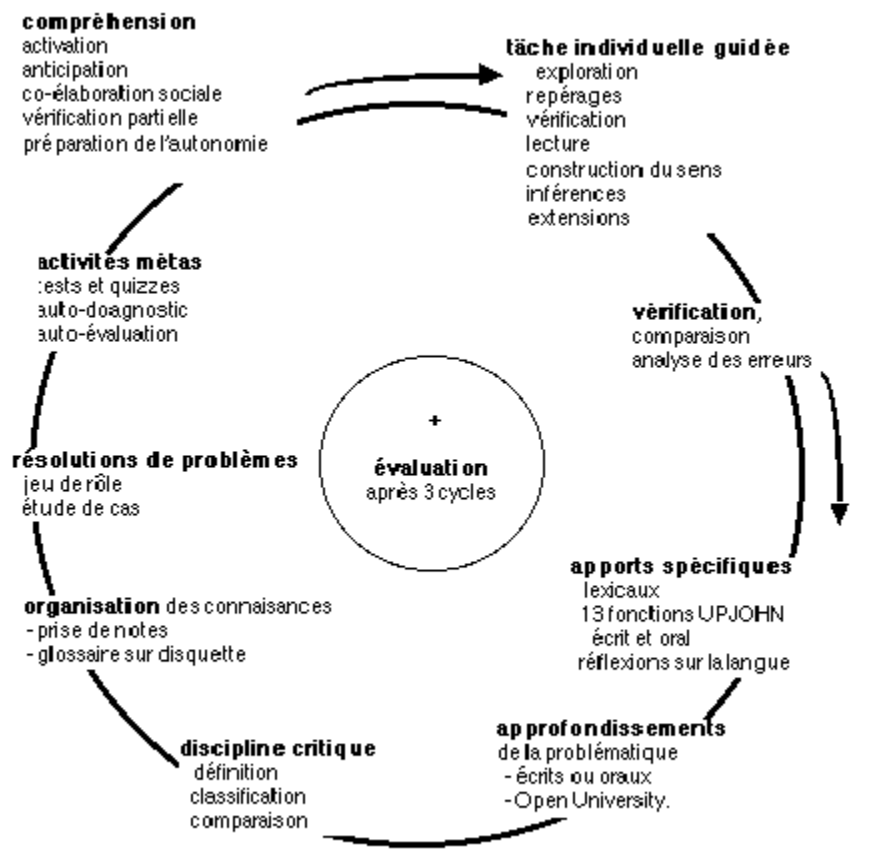

\section{NOTES}

1. VIFAX fonctionne depuis mars 1990 à partir de l'Université Victor-Segalen Bordeaux 2. Les établissements abonnés enregistrent sur vidéocassette tous les matins les informations télévisées de chaînes britannique ou américaine (VIFAX existe aussi pour l'allemand et pour le français langue étrangère). À partir de midi, ils reçoivent par fax, ou par fichier annexé à un courrier électronique une batterie d'exercices, leur corrigé, et la transcription intégrale des deux séquences traitées quotidiennement. Les tâches à accomplir sont conçues pour entraîner un effort cognitif qui permet à chacun de construire au jour le jour une vraie compétence de compréhension de l'anglais réel, avec l'ouverture culturelle que procurent les informations télévisées, et, naturellement, l'intérêt de l'immédiateté. Bien entendu, le travail peut donner lieu à exploitation, et surtout prolongements en classe sous la conduite du professeur : le système ACADEME qu'a mis au point Jean Sabiron à Poitiers en est un excellent exemple [NDLR].

2. LANSAD : le secteur des "Langues pour spécialistes d'autres disciplines » : acronyme proposé par M. Perrin au colloque 1993 d'Aix-en-Provence sur l'enseignement des langues aux... nonspécialistes, précisément pour tenter de tordre le cou à cette dernière appellation dévalorisante. 


\section{RÉSUMÉS}

Par une combinatoire d'outils langagiers adaptés aux goûts et besoins des étudiants de sciences, élaborés régulièrement sous le nom d'ACADEME, on parvient à faire de l'enseignement de l'anglais à ces « non-spécialistes » l'instrument d'une vraie progression intellectuelle et humaine.

By providing science students with integrated, custom-made tools for language learning (the system has been called ACADEME), the compulsory teaching of English to non-linguists has become an opportunity for them to mature intellectually and as human beings.

\section{INDEX}

Mots-clés : cohérence, construction du savoir, éclectisme, outil langagier, visée intégrative Keywords : construction of knowledge, eclecticism, integrative aim, language tool

\section{AUTEUR}

\section{JEAN SABIRON}

Après avoir soutenu à Bordeaux sa thèse « Langue anglaise et étudiants scientifiques : une combinatoire d'outils langagiers et méthodologiques en vue du perfectionnement de la compréhension de l'anglais oral, autour d'un centre de ressources, le concept d'ACADEME ", Jean Sabiron est depuis septembre 1996 maître de conférences à la faculté des lettres de l'université de Poitiers. jean.sabiron@wanadoo.fr 